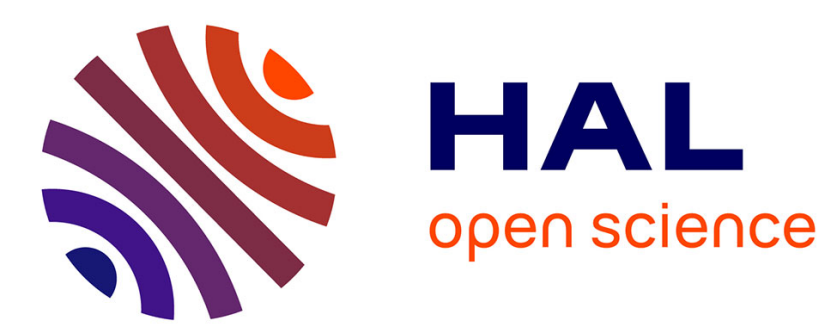

\title{
The Ordered Timeline Game: Strategic Posting Times Over a Temporally Ordered Shared Medium
}

\author{
Eitan Altman, Nahum Shimkin
}

\section{To cite this version:}

Eitan Altman, Nahum Shimkin. The Ordered Timeline Game: Strategic Posting Times Over a Temporally Ordered Shared Medium. Dynamic Games and Applications, 2015, pp.1-25. 10.1007/s13235015-0158-y . hal-01152461

\section{HAL Id: hal-01152461 \\ https://hal.inria.fr/hal-01152461}

Submitted on 17 May 2015

HAL is a multi-disciplinary open access archive for the deposit and dissemination of scientific research documents, whether they are published or not. The documents may come from teaching and research institutions in France or abroad, or from public or private research centers.
L'archive ouverte pluridisciplinaire HAL, est destinée au dépôt et à la diffusion de documents scientifiques de niveau recherche, publiés ou non, émanant des établissements d'enseignement et de recherche français ou étrangers, des laboratoires publics ou privés. 


\title{
Strategic Choices of Posting Time of Content over a Temporally-Ordered Shared Medium
}

\author{
Eitan Altman ${ }^{1}$ and Nahum Shimkin ${ }^{2}$ \\ ${ }^{1}$ INRIA, 2004 Route des Lucioles, 06902 Sophia-Antipolis Cedex, France; \\ ${ }^{2}$ Electrical Engineering Dept., Technion, Haifa 32000, Israel
}

\begin{abstract}
We consider a game of timing between a random number of content creators, who compete for position and exposure time over a shared medium such as an on-line classified list. Contents (such as ads, messages, multimedia items or comments) are ordered according to their submission times, with more recent submissions displayed at the top (and better) positions. The instantaneous effectiveness of each ad depends on its current display position, as well as on a time-dependent exposure function common to all. Each content creator may choose the submission time of her content within a finite time interval, with the goal of maximizing the total exposure of this content. We formulate the problem as a non-cooperative game, analyze its symmetric equilibrium, characterize it in terms of a differential boundary value problem and devise a numerical schemes for its computation.
\end{abstract}

\section{Introduction}

Consider an online media site, or display board, to which each of several players posts one content such as an advertisement, news item, online comment, a picture or a video clip. Each player chooses the time to post her content. Posted contents are positioned on the board in descending order according to their arrival time, with the latest item displayed at the top (best) position, and is gradually moved to lower positions (and eventually removed) as new items arrive and take its place. Each player is interested in maximizing the exposure, or effectiveness, of his own posted content. A couple of time-related factors affect this effectiveness (in addition to the appeal of the content). One is the overall exposure of the site, or viewer potential, which in general depends on time (e.g., on the time of day). The second is the presumed rate of postings by other players, which may lead to closely spaced posts that compete for the viewers' attention. In our model, taking the view point of a specific player, each new post that appears after our own lowers our position on the display board, leading to lower exposure potential. The latter interaction between the players leads to a noncooperative game of timing, which we analyze in this paper.

There are of course many other situations that present a similar timing tradeoff between peak demand periods and the level of peer competition. These may range from finding the best time to share a link on a social network, to marketing oriented decisions of choosing the time to release a new product or launch 
a publicity campaign. See, for example, the discussions in $[17,16,3]$ regarding the seasonality of demand and new product introduction in the food market and in the motion picture industry, and the game formulation of these timing problems in $[12,11]$. In the online advertising context, practical guides address the reduction of ad bid prices by avoiding periods of high advertiser competition [1].

We assume that the instantaneous effectiveness (or exposure) of a displayed item is determined by its position in the board (with higher positions considered better), modulated by a time-dependent site exposure function $u(t)$ that represents the expected traffic or interest in the site at time $t$. The number of players is random with Poisson distribution. The number of players is not known but its distribution is common knowledge. Each participating player choose independently the submission time of his content, and aims to maximize the cumulative exposure of this item.

Related literature: The closet work to ours in the is paper [15], which considered a similar game model with a single board position. andn a fixed known numberof players. The symmetric equilibrium was explicitly computed for the two-player game, and a discretization scheme was proposed for computing an $\epsilon$ optimal equilibrium for the $n$-player case. Our model differs from [15] in several respects:

- A time-varying exposure function $u(t)$ is considered, rather than a constant.

- We allow for multiple board positions, rather than a single one.

- We consider a random number of participating players.

Other timing games have been considered in the literature. In such games, sole action of each player is a stopping time. Some classical two-player games of timing, which include the the War of Attrition and preemption game, are reviewed in [4], Chapter 4.5; more recent pointers may be found in [13]. In the transportation literature, Vickrey's bottleneck model [18] addresses strategic timing decisions of commuters who balance road congestion delay with late or early arrival to their destination, where congestion is modeled as a fluid queue. This model has been extensively studied and extended in various directions - see, e.g., $[14,9]$ and their references. A similar model which incorporates a stochastic queue with exponentially distributed service times was introduced in [5], and further extended recently in $[7,10,8]$. It is interesting to note that in these papers, since the players are lined up in a First Come First Served queue, only players that arrive before us affect our payoff. In the present model the opposite is true - only players that arrive after us affect our payoff.

Main results: For our general model, we characterize the symmetric equilibrium point and provide a numeric procedure for its computation. It is first shown that the equilibrium strategy of each player is mixed, namely a probability distribution over the game interval $[0, T]$, which is continuous and supported on a sub-interval $[0, L]$. We refer to that distribution as the equilibrium profile. It is characterized in terms of an integral equation, or equivalently in terms of a functional differential equation (FDE) that evolves backward in time. Using the 
latter characterization we establish existence of an equilibrium profile, and provide a numeric procedure that involves a single-parameter search for computing all symmetric equilibria. We also provide a sufficient condition for uniqueness of the symmetric equilibrium, in terms of a certain convexity requirement on the relative utility parameters of the different board positions. We also provide an expression for the social efficiency of the equilibrium solution, namely the under-usage of the board in equilibrium relative to its full potential occupancy.

The remainder of the paper is organized as follows. Section 2 presents the system and game model. Section 3 presents our main results for the general model, including existence, uniqueness, structure, and computation of the equilibrium profile. The proof of these results are collected in Section 3. Sections 4 and 5 discuss the special cases of Poisson demand and two-player game, respectively, while Section 6 presents some numerical results. We conclude the paper in Section 7.

\section{Model Description}

We consider an on-line display or advertising board, on which users (or players) can post their items during a given time interval $[0, T]$. The board consists of $K \geq 1$ positions, where position 1 is the most effective, and last position $K$ the least effective. The position of the posted items on the board is dynamically determined according to their order of arrival: a newly arrived item is placed at the top position, while existing items are pushed one position lower (from 1 to 2,2 to 3 etc.). The item at position $K$ (if any) is ejected dropped the board.

For $t \in[0, T]$ and $k=1, \ldots, K$, let $u_{k}(t)$ denote the expected utility rate (per unit time) for a displayed item at time $t$ in position $k$. The total expected utility over the entire life cycle of an item is therefore

$$
U\left(\mathcal{T}_{1}, \ldots, \mathcal{T}_{K}\right)=\sum_{k=1}^{K} \int_{t \in \mathcal{T}_{k}} u_{k}(t) d t,
$$

where $\mathcal{T}_{k}$ is the time interval on which the item was displayed at position $k$. We assume that the functions $u_{k}(t)$ are decomposed as

$$
u_{k}(t)=r_{k} u(t), \quad t \in[0, T], k=1, \ldots, K,
$$

where

- The exposure function $u(t)$, which is common to all positions, captures the the temporal dependence of the utility, due to variation in the exposure of the entire board.

- The constants $\left(r_{k}, k=1, \ldots, K\right)$ are the relative utility parameters, which capture the relative effectiveness of the different positions in the board. That is, the relative utilities are positive, and are decreasing in the board position $k$. It will be convenient for our analysis to define $r_{k}=0$ for $k \geq K+1$.

The following assumptions are imposed throughout the paper. 


\section{Assumption 1}

(i) The exposure function $u:[0, T] \rightarrow \mathbb{R}$ is continuous and strictly positive, namely $u(t)>0$ for $t \in[0, T]$. Let $u_{\min }>0$ and $u_{\max }$ denote the extremal values of $u$.

(ii) The relative utility parameters $\left(r_{k}\right)$ are decreasing in the board position. Specifically,

$$
r_{1}>r_{2} \geq r_{3} \cdots \geq r_{K}>0
$$

The game formulation involves several players, who compete for a place in the board and wish to to maximize their individual utilities. Each player $i$ chooses the submission time $t_{i}$ of his own item. As mentioned, upon submission, the item is placed in the top position, but goes down in rank as further items are posted. Clearly, the utility of each player depends on his or her own choice of submission time $\left(t_{i}\right)$, as well as the submission times of the other player. We therefore consider the problem as a non-cooperative game, and analyze the Nash equilibrium of this game.

To complete the game description, we specify some additional properties.

1. The number of players who participate in a given instance of the game is a random variable, denoted $D_{0}$. We refer to $D_{0}$ as the objective demand.

2. The belief of each participating player regarding the number of other players in the game is another random variable, denoted $D$. We refer to $D$ as the subjective demand. Clearly, if $D_{0}$ is deterministic then $D=D_{0}-1$. The general relation between $D$ and $D_{0}$ is discussed in Subsection 2.3. Let $p_{D}=$ $\left(p_{D}(n), n \geq 0\right)$ denote the distribution of $D$. We assume that $E(D)<\infty$, and further, to avoid triviality, that $r_{D+1}<r_{1}$ with positive probability ${ }^{\star}$.

3. A player cannot observe the submission times of others before choosing his own submission time; in particular, the players do not observe the board status before their arrival. (As we shall see, the latter assumption can be relaxed when $D$ follows a Poisson distribution.)

4. The submission time $t_{i}$ of player $i$ can be chosen randomly, according to a probability distribution on $[0, T]$ with cumulative distribution function $F_{i}(t), t \in[0, T]$. The corresponding density function, when it exists, will be denoted by $f_{i}(t)$. We refer to $F_{i}$ as the (mixed) strategy of player $i$.

We shall be interested in the Nash equilibrium point (NEP) of this game. Specifically, we consider the symmetric NEP in which the strategies of all players are identical, namely $F_{i} \equiv F$ all players. The restriction to symmetric strategies, besides its analytical tractability, seems natural in the present scenario where players are essentially anonymous. We proceed to calculate the players' utilities for the symmetric case.

\footnotetext{
* Otherwise, all $D+1$ players can arrive at $t=0$ and remain in positions with maximal relative utility $r_{1}$ all the way up to $T$.
} 


\subsection{Expected Utility}

Consider a certain player $i$ who posts his item at time $t$. Suppose that each of the other $D$ players uses an identical strategy $F$. We proceed to calculate the expected utility $U(t ; F)$ of the player in that case.

Suppose first that $F$ has no point mass at $t$, so that with probability 1 there are no simultaneous arrivals at $t$. Let $N_{(t, s]}$ denote the number of arrivals (by other players) during the time interval $(t, s]$, for $t<s \leq T$. Since $i$ arrives at $t$, his position in the board at time $s$ will be $k+1$ if $N_{(t, s]}=k$, for $0 \leq k \leq K-1$, and he would have left the board if $N_{(t, s]} \geq K$. It follows that

$$
\begin{aligned}
U(t ; F) & =\mathbb{E}_{F}\left(\int_{t}^{T} \sum_{k=0}^{K-1} r_{k+1} \mathbf{1}_{\left\{N_{(t, s]}=k\right\}} u(s) d s\right) \\
& =\int_{t}^{T} \sum_{k=0}^{K-1} r_{k+1} \mathbb{P}_{F}\left(N_{(t, s]}=k\right) u(s) d s .
\end{aligned}
$$

To compute the probability $\mathbb{P}_{F}\left(N_{(t, s]}=k\right)$, recall that the number of participating players other than $i$ is a random variable $D$. The probability that each of these players submits his item on $(s, t]$ is $F(s)-F(t)$. Therefore, conditioned on $D=n, N_{(t, s]}$ follows a Binomial distribution $\operatorname{Bin}(n, p)$ with success probability $p=F(s)-F(t)$. Denoting

$$
B_{k, n}(p)=\left(\begin{array}{l}
n \\
k
\end{array}\right) p^{k}(1-p)^{n-k}, \quad 0 \leq k \leq n
$$

(and setting $B_{k, n} \equiv 0$ for $k>n$ ), we obtain

$$
\begin{aligned}
\mathbb{P}_{F}\left(N_{(t, s]}=k\right) & =\sum_{n \geq k} p_{D}(n) \mathbb{P}_{F}\left(N_{(t, s]}=k \mid D=n\right) \\
& =\sum_{n \geq k} p_{D}(n) B_{k, n}(F(s)-F(t)) .
\end{aligned}
$$

Substituting (3) in (1) gives

$$
U(t ; F)=\int_{t}^{T} \sum_{k=0}^{K-1} r_{k+1} \sum_{n \geq k} p_{D}(n) B_{k, n}(F(s)-F(t)) u(s) d s .
$$

We mention some special cases of this expression.

Single Board Position: When $B=1$, noting that $B_{0, n}(p)=(1-p)^{n}$ we obtain

$$
U(t ; F)=r_{1} \int_{t}^{T} \sum_{n \geq 0} p_{D}(n)(1-F(s)+F(t))^{n} u(s) d s .
$$

Deterministic Demand: Suppose $D$ is a deterministic positive integer (which corresponds to a game with $D_{0}=D+1$ players). In that case

$$
\mathbb{P}_{F}\left(N_{(t, s]}=k\right)=B_{k, D}(F(s)-F(t)),
$$


and

$$
U(t ; F)=\int_{t}^{T} \sum_{k=0}^{K-1} r_{k+1} B_{k, D}(F(s)-F(t)) u(s) d s .
$$

Poisson Demand: Suppose $D \sim \operatorname{Pois}(\Lambda)$, a Poisson random variable (RV) with parameter $\Lambda>0$, namely $p_{D}(n)=\Lambda^{n} e^{-\Lambda} / n$ ! for $n \geq 0$. Since a Bernoulli dilution of a Possion RV remains Poisson, it follows that $N_{(t, s]}$ is a Poisson RV with parameter $\Lambda(F(s)-F(t))$, and

$$
\mathbb{P}_{F}\left(N_{(t, s]}=k\right)=\frac{1}{k !} \Lambda^{k}(F(s)-F(t))^{k} e^{-\Lambda(F(s)-F(t))} .
$$

This expression can be directly substituted in Equation (1).

Simultaneous arrivals: If $F$ has a point mass at $t$, then there is a positive probability of simultaneous arrivals of several players at that time. In that case we assume that their order of arrival (and subsequent positioning on the board) is determined uniformly at random. The utility $U(t ; F)$ needs to be modified accordingly. We need not bother here with writing the straightforward but cumbersome expression, as we argue in Lemma 1 below that in equilibrium $F$ does not possess point masses.

\subsection{Nash Equilibrium}

A symmetric Nash equilibrium point (NEP) is represented by a strategy $F$, which is a probability distribution on $[0, T]$, such that $F$ is a best response for each player when all others use the same strategy $F$. More formally, for any pair of strategies $G$ and $F$, let

$$
\bar{U}(G ; F)=\mathbb{E}_{t \sim G}(U(t ; F))=\int_{t} U(t ; F) d G(t)
$$

denote the expected utility of a player for using strategy $G$ when all others follow $F$. Then $F$ represents a symmetric NEP if

$$
F \in \underset{G}{\operatorname{argmax}} \bar{U}(G ; F),
$$

where the maximum is taken over all probability distributions on $[0, T]$. We shall refer to a symmetric equilibrium strategy $F$ as an equilibrium profile.

An equivalent definition of the symmetric NEP, that is more useful for the analysis, requires $U(t ; F)$ to be minimized on a set of times $t$ of $F$-probability 1 . That is:

There exists a constant $u^{*}$ and a set $A \subset[0, T]$, such that $\int_{A} d F(t)=1$, and

$$
\begin{gathered}
U(t ; F)=u^{*} \text { for } t \in A, \\
U(t ; F) \leq u^{*} \text { for } t \notin A .
\end{gathered}
$$

The equivalence of the two definitions is readily verified. We refer to the constant $u^{*}=u_{F}^{*}$ as the equilibrium utility corresponding to an equilibrium profile $F$. 


\subsection{Demand Distributions}

Recall that the random variable $D$ stands for the subjective demand, which represents the belief of a participating player regarding the number of other participating players, while the random variable $D_{0}$ stands for the objective demand, namely the number of players that participate in a given instance of the game. The objective load $D_{0}$ does not play a direct role in determining the equilibrium profile; it is required however for determining social utility, and of course influences $D$.

A well known formula from [2] connects the distributions of $D$ and $D_{0}$ under symmetry conditions. Let $p_{0}(n)=P\left(D_{0}=n\right)$ denote the distribution of $D_{0}$. We are interested in the conditional probabilities $P(D=n-1)=P\left(D_{0}=n \mid A_{i}\right)$, where $A_{i}$ is the event that a certain player $i$ participates in the game. Then

$$
P(D=n-1)=\frac{1}{c} n p_{0}(n), \quad n \geq 1
$$

where $c=\sum_{n>1} n p_{0}(n)$ is the normalization constant. The argument that leads to (7) is roughly as follows. Suppose that the $D_{0}$ participants are selected symmetrically from a finite population of size $N$. By symmetry, the probability of a particular subset $B$ of the population to be chosen depends only on its size $|B|$. By comparing the number of subsets of size $n$ in which a given player $i$ is selected to the total number of such subsets, we obtain:

$$
p\left(A_{i} \mid D_{0}=n\right)=\left(\begin{array}{c}
N-1 \\
n-1
\end{array}\right) /\left(\begin{array}{c}
N \\
n
\end{array}\right)=\frac{n}{N} .
$$

Invoking Bayes formula now leads to (7).

The relation (7) should be used with some caution. It relies on the assumption that participation decisions are fully symmetric, and that the potential participants know exactly the distribution of $D_{0}$. Furthermore, the significant skewness (by a factor $n$ ) in the distribution of $D$ in (7) may be attributed to correlation in the joining decisions of the players. However, if this correlation is induced by a public signal, then the players can actually condition on that signal before choosing their strategy.

Rather than dealing further with the general case, when considering specific demand distributions we will focus on two special cases where the abovementioned correlation is absent. The first case is the deterministic case, $D_{0}=n$, for which indeed $D=n-1$ according to (7). The second case is the Poisson distribution, $D_{0} \sim \operatorname{Poiss}(\Lambda)$, for which we obtain from $(7)$ that $D \sim \operatorname{Poiss}(\Lambda)$, i.e., the same distribution as $D_{0}$.

We note that both these cases can be viewed as extremes of the Binomial distribution, where $n$ potential players decide independently whether to join or not. For $D_{0} \sim \operatorname{Bin}(n, p)$, we obtain $D \sim \operatorname{Bin}(n-1, p)$. The deterministic case is obtained for $p=1$, while the Poisson case can be viewed as the limit of taking $n \rightarrow \infty$ while keeping $n p=\Lambda$. 


\section{Equilibrium Analysis}

We present in this section the main properties of the equilibrium profile that apply to the general model. We first establish existence, as well as some useful structural properties that include characterization of the equilibrium distribution in terms of a functional differential equation, and a computational procedure that relies on this equation. We then present sufficient conditions for uniqueness of the equilibrium, and finally discuss the social efficiency of the equilibrium. The proofs of these results are presented in the next section.

\subsection{Existence and Characterization}

For an arrival profile $F$ and $t \in[0, T]$, denote

$$
g(t, F)=\sum_{k=0}^{K-1}\left(r_{k+1}-r_{k+2}\right) \sum_{n \geq k}(n+1) p_{D}(n+1) \int_{t}^{T} B_{k, n}(F(s)-F(t)) u(s) d s
$$

Let $F^{\prime}(t)$ denote the time derivative of $F$ at $t$. Recall that the support of a probability measure $\eta$ is the smallest closed set of $\eta$-probability 1 . For brevity, we denote by $\operatorname{supp}(F)$ the support of the probability measure $\eta_{F}$ induced by a distribution function $F$. Finally, recall that $U(t ; F)$ is the expected utility which is specified in (4).

Theorem 1 (Existence and Characterization). An equilibrium profile $F=$ $(F(t), t \in[0, T])$ exists. Any equilibrium profile satisfies the following properties.

(i) $F$ is a continuous function, and there exists a number $L \in(0, T)$ such that $\operatorname{supp}(F)=[0, L]$. Specifically, $F(0)=0, F(L)=1$, and $F(t)$ is strictly increasing in $t \in[0, L]$.

(ii) Consequently, a continuous probability distribution function $F$ on $[0, T]$ is an equilibrium profile if, and only if, there exists a number $L \in(0, T)$ such that $F(0)=0, F(L)=1$, and $U(t ; F)=u_{L}$ for $t \in[0, L]$ and some constant $u_{L}>0$.

(iii) Equivalently, a continuous probability distribution function $F$ on $[0, T]$ is an equilibrium profile if, and only if, there exists a number $L \in(0, T)$ such that: $F(0)=0, F(L)=1$, and the derivative $F^{\prime}(t)$ exists for $t \in(0, L)$ and satisfies the equality

$$
F^{\prime}(t)=\frac{r_{1} u(t)}{g(t, F)}, \quad t \in(0, L)
$$

where $g(t, F)$ is defined in (8).

(iv) For an equilibrium profile $F$ with support $[0, L]$, the equilibrium utility $u_{F}^{*}$ is given by $u_{F}^{*}=r_{1} \int_{L}^{T} u(s) d s$. 
Proof outline: The full proof is presented Appendix A. Here we provide and brief outline, for the reader's benefit.

(i) To argue that $F$ is continuous, suppose (see Lemma $1(i)$ ) that $F$ has an upward jump (i.e., a point mass) at $t$. This means that there is a positive chance that several ads are submitted simultaneously at $t$. But then it would be better to submit the ad just after $t$, leading to $U(t ; F)<U\left(t^{+} ; F\right)$, which contradicts the assumption that $t$ is in $\operatorname{supp}(F)$.

To show that $\operatorname{supp}(F)$ is an interval $[0, L]$, suppose by contradiction that there exists a gap in that support, namely numbers $0 \leq a<b$ such that $F(a)=$ $F(b)<1$. We may extend $b$ to the right till it hits $\operatorname{supp}(F)$. But since there are no submissions in $(a, b)$, it follows that $U(a ; F)<U(b ; F)$, which means that $b$ cannot belong to the support $\operatorname{supp}(F)$; see Lemma 1(iii).

(ii) If $F$ is an equilibrium profile then $\operatorname{supp}(F)=[0, L]$ by $(i)$, and $U(t ; F)=c$ on $t \in[0, L]$ follows by definition of the equilibrium and the continuity of $U(t ; F)$ in $t$ (Lemma $1(i i)$ ). The converse follows by noting that if $F(L)=1$, then there are no arrivals on $(L, T]$ which implies that $U(t ; F)>U(L ; F)=u_{L}$ for $t>L$.

(iii) Differentiating the expression (4) for $U(t ; F)$ gives $\frac{d}{d t} U(t ; F)=-r_{1} u(t)+$ $F^{\prime}(t) g(t, F)$ (Lemma 3). Now, if $F$ is an equilibrium profile then $U(t ; F)$ is constant on $[0, L]$, then $\frac{d}{d t} U(t ; F)=0$ on $(0, L)$, and we obtain the differential relation (9). The converse statement follows by arguing that (9) implies that $U(t ; F)$ is constant on $[0, L]$; see Lemma 4 .

Part (iv) of the Theorem is straightforward, upon noting that there are no new submissions on $(L, T]$ so that an ad submitted at $t=L$ remains at the top position till $T$.

The proof of existence of an equilibrium relies on the differential characterization in part (iii). For that purpose, we consider Equation (9) as a (functional) differential equation with terminal conditions $F(t)=1$ for $t \in[L, T]$. It is argued that the solutions $F_{L}(t)$ to this equation are well defined and continuous in $L$ (Proposition 1). From this we deduce (Lemma 5 ) that $F_{L}(0)=0$ for some $L$, which implies that the corresponding solution $F_{L}(t)$ is an equilibrium profile.

\section{Remarks}

1. Note that property (iii) of the Theorem implies that $F$ admits a density $f$, which is strictly positive on $[0, L)$ and zero on $(L, T]$.

2. The differential relation in (9) provides a functional differential equation for $F$, as the right-hand side depends on entire function $F$ and not only on its value at $t$. This relation provides the basis for the existence and uniqueness claims in this and the next subsection, as well as for the computational procedure that follows.

3. The results of the last Theorem do not imply uniqueness of the equilibrium profile. However, if multiple equilibria do exist, we obtain a strict ordering among them. First, we note that to each number $L$ and support $[0, L]$, there correspond at most one equilibrium profile (this follows by uniqueness of solutions to Equation (9), see Proposition 1 below). Consider now two equilibrium profiles $F_{1}$ and $F_{2}$, indexed by $L_{1}$ and $L_{2}$ respectively, with $L_{1}<L_{2}$. By part (iv) of the Theorem it follows that the equilibrium utility $u^{*}$ of $F_{1}$ 
is strictly higher than that of $F_{2}$. That is: the equilibrium with the smaller support $[0, L]$ is better (in terms of individual utilities).

\section{$3.2 \quad$ Uniqueness}

To establish uniqueness of the equilibrium profile, we require an additional condition on the relative utilities $\left(r_{k}\right)$. We observe that this is only a sufficient condition, and uniqueness may well hold in greater generality.

Assumption 2 The relative utility parameters satisfy the following convexity condition:

$$
r_{k} \leq \frac{1}{2}\left(r_{k-1}+r_{k+1}\right), \quad k=2, \ldots, K
$$

(recall that $r_{K+1}=0$ by definition).

Theorem 2 (Uniqueness). Suppose Assumption 2 holds, then the equilibrium profile $F$ is unique.

We note that Assumption 2 holds trivially for a single board position, namely $K=1$. It also holds for linearly-decreasing utilities of the form $r_{1}=(K+$ 1) $d, \ldots, r_{K}=d$, for some $d>0$, as well as for geometrically decreasing utilities: $r_{k}=\gamma^{k}, 1 \leq k \leq k_{0}$ for $\gamma \in(0,1)$ and $k_{0} \leq K$ (if $\gamma \leq 0.5$ we can take $k_{0}=K$; otherwise we need to continue $r_{k}$ beyond $k_{0}$, say linearly, so that the convexity condition holds up to $r_{K+1}=0$ ). The assumption does not hold in the interesting case where $K \geq 2$ and some board positions have equal weights, say $r_{1}=r_{2}$.

\subsection{Computation}

We outline next a numeric procedure for computing all equilibrium profiles. This procedure relies on computing the solution $F$ of the functional differential equation (FDE) (9) for different values of the parameter $L \in(0, T)$, and searching for values of $L$ for which the boundary conditions $F(L)=1$ and $F(0)=0$ are satisfied. The latter essentially involves an exhaustive search over the scalar parameter $L$. When the uniqueness condition in Assumption 2 is satisfied, that search can be expedited by observing monotonicity properties of the solution $F$ in $L$.

For each $L \in(0, T)$, we consider the differential equation (9), with terminal conditions $F(t)=1, t \in[L, T]$. As mentioned, this is a functional differential equation since the derivative $F^{\prime}(t)$ at timet depends on values of $F$ at other times as well. However, a key property of that equation, which follows from the definition of $g(t, F)$ in (8), is that this dependence is one-sided: $F^{\prime}(t)$ depends only on 'future' values of $F$, namely on $(F(s), s \geq t)$. In the terminology of [6], this equation is a retarded FDE (up to time reversal). This property allows to backintegrate this equation, starting with the above-mentioned terminal conditions, and proceeding backward in time.

We collect some properties of the FDE (9) in the following Proposition. These support the numerical computation procedure that follows, and are also used in the proofs of the pervious results. 
Proposition 1. For $L \in(0, T)$, define $F_{L}(t)=1$ for $t \in[L, T]$. Consider the FDE (9) for $F_{L}$, namely

$$
F_{L}^{\prime}(t)=\frac{r_{1} u(t)}{g\left(t, F_{L}\right)}, \quad t \leq L
$$

where $g$ is defined in (8).

(i) The FDE (11) admits a unique solution $F_{L}(t)$ over $t \in\left[t_{L}, L\right]$, where

$$
t_{L}=\inf \left\{s \in[0, L): F_{L}(s)>0\right\} .
$$

(ii) There exists a positive constant $\epsilon_{L}>0$ such that $g\left(t, F_{L}\right) \geq \epsilon_{L}$ for all $t \in$ $\left[t_{L}, L\right]$. Consequently, $F_{L}^{\prime}(t) \leq\left(r_{1} u_{\max } / \epsilon_{L}\right)<\infty$ for $t \in\left[t_{L}, L\right]$.

(iii) $t_{L}$ is a continuous function of $L$, and $F_{L}(t)$ is a continuous function of $L$ for each $t \in\left(t_{L}, L\right]$.

Note that $t_{L}$ is the first time $t \geq 0$ at which $F_{L}(t)$ becomes zero. As $g\left(t, F_{L}\right)$ is meaningless for $F_{L}<0$, the solution of equation (11) cannot be extended beyond this point. For the sake of exposition, it will be convenient to linearly extend $F_{L}(t)$ below $t_{L}$ when $t_{L}>0$, using

$$
F_{L}(t)=-\left(t_{L}-t\right), \quad t \in\left[0, t_{L}\right] .
$$

This gives $F_{L}(0)<0$ when $t_{L}>0$.

The equilibrium profiles now correspond to those values of $L$ for which $F_{L}(0)=0$. To search for these values, we may use the following crude exhaustive search approach:

- For values of $L$ in a grid over $(0, T)$, integrate equation (11) numerically (using Euler approximation or a more accurate method) and obtain $F_{L}(t)$, $t \in[0, T]$.

- The equilibrium points correspond to values of $L$ where $F_{L}(0) \simeq 0$.

The search grid may of course be refines around points of interest. We note that under the uniqueness condition (10), we know that $F_{L}(0)$ crosses 0 at a unique value of $L$, which clearly simplifies the search.

It should be possible to compute explicit bounds on the variation of $F_{L}(t)$ in $L$, which may lead to formal guarantees on $\epsilon$-optimality of computed equilibrium profile. This is however beyond the scope of he present paper. We merely point out here that the level of sub-optimality of any candidate equilibrium profile $F$ can be easily tested numerically by computing the utility function $U(t ; F)$ in (4) and evaluating its deviation from a constant over $[0, L]$.

\section{Remarks}

1. We observe that if $F(t)$ is an equilibrium profile for the model with the given exposure function $u(t)$, then $\tilde{F}(t)=F(g(t))$ is an equilibrium profile for the model with unit exposure function, $u(t) \equiv 1$, where $g(t)$ denotes the time change

$$
g(t)=\frac{T}{\int_{0}^{T} u(s) d s} \int_{0}^{t} u(s) d s .
$$


This follows by verifying, through a change of integration variable, that $U(t ; F)$ in equation (4) is equal to $U(g(t), \tilde{F})$ in the model with $u=1$. Therefore, once the equilibrium profile is computed for one exposure function $u(t)$, it can be readily obtained for any other.

2. Our standing Assumption 1 requires a strict inequality in $r_{1}<r_{2}$. If this is violated, namely $K \geq 2$ and $r_{1}=r_{2}$, it may be seen that $g\left(L, F_{L}\right)=0$, which leads to a difficulties in initializing the backward solution of equation (11). A direct solution therefore requires more elaborate numeric schemes than the ones considered here. Rather than go into these, this case may be handled by solving an approximate problem with $r_{1}=r_{2}+\epsilon$, for $\epsilon$ small enough.

\subsection{Social Efficiency}

Under an equilibrium profile $F$ with support $\operatorname{supp}(F)=[0, L]$, the expected utility $u_{F}^{*}$ per player is given by Theorem $1(i v)$. Recalling that $D_{0}$ is the subjective demand, the expected social utility may be seen to be

$$
S(F)=E\left(D_{0}\right) u_{F}^{*}=E\left(D_{0}\right) r_{1} \int_{L}^{T} u(s) d s .
$$

Let us compare that to the optimal social utility. Under full utilization, namely all $K$ positions occupied over $[0, T]$, the social utility is clearly

$$
\sum_{k=1}^{K} r_{k} \int_{s=0}^{T} u(s) d s
$$

However, taking into account that if $n<k$ player arrive then some lower positions remain empty, the optimal social utility becomes

$$
S^{*}=\sum_{k=1}^{K} r_{k}\left(\sum_{n \geq k} p_{0}(n)\right) \int_{s=0}^{T} u(s) d s
$$

The relative efficiency of the equilibrium is now defined and evaluated by the ratio:

$$
\rho(F) \triangleq \frac{S(F)}{S^{*}}=\frac{r_{1} E\left(D_{0}\right)}{\sum_{k=1}^{K} r_{k} \sum_{n \geq k} p_{0}(n)} \frac{\int_{L}^{T} u(s) d s}{\int_{0}^{T} u(s) d s} .
$$

This expression still depends on the equilibrium parameter $L$. Explicit expressions for some specific cases will be derived in the following sections.

\section{Special Case I: Poisson Demand}

We consider in this section the special case of Poisson demand. After discussing some general relations, we further specialize to the case of a board position, 
namely $K=1$. We will also consider formally the more general model of an infinite board with geometrically decreasing utility parameters. In both cases we provide explicit formulae for the equilibrium profile, as well as for the associated efficiency loss.

Suppose, then, that system demand $D_{0}$ is a Poisson random variable with parameter $\Lambda>0$, namely $D_{0} \sim \operatorname{Pois}(\Lambda)$, and $p_{0}(n)=\Lambda^{n} e^{-\Lambda} / n$ ! for $n \geq 0$.

As mentioned in the Introduction, the Poisson assumption models the case where the participating players are chosen randomly and independently from a large population of potential participants. Also, as mentioned above, a unique property of the Poisson demand is that the subjective and objective demand distributions, related by (7), are equal. Hence, $D \sim \operatorname{Pois}(\Lambda)$, and $p_{D}(n)=\Lambda^{n} e^{-\Lambda} / n$ ! for $n \geq 0$.

The utility function $U(t ; F)$ can now be evaluated by (4), using some algebra. More directly, since Bernoulli dilutions of a Poisson random variable remain Poisson, it follow that $N_{(t, s]}$ in (1) is a Poisson random variable with parameter $\Lambda(F(s)-F(t))$, and

$$
\mathbb{P}_{F}\left(N_{(t, s]}=k\right)=\frac{1}{k !} \Lambda^{k}(F(s)-F(t))^{k} e^{-\Lambda(F(s)-F(t))} .
$$

Therefore,

$$
U(t ; F)=\int_{t}^{T} \sum_{k=0}^{K-1} r_{k+1} \frac{1}{k !} \Lambda^{k}(F(s)-F(t))^{k} e^{-\Lambda(F(s)-F(t))} u(s) d s
$$

\subsection{Unit Board}

Consider now the case of a single board position, $K=1$, with utility function $u_{1}(t)=r_{1} u(t)$. From (14) we obtain

$$
U(t ; F)=r_{1} \int_{t}^{T} e^{-\Lambda(F(s)-F(t))} u(s) d s
$$

We proceed to derive (9) directly. By differentiating the last expression,

$$
\frac{d}{d t} U(t ; F)=-r_{1} u(t)+r_{1} \Lambda F^{\prime}(t) \int_{t}^{T} u(s) e^{-\Lambda[F(s)-F(t)]} d s .
$$

Since $\frac{d}{d t} U(t ; F)=0$ on $(0, L)$, we obtain

$$
F^{\prime}(t)=\frac{r_{1} u(t)}{\Lambda r_{1} \int_{t}^{T} e^{-\Lambda[F(s)-F(t)]} u(s) d s}, \quad t \in(0, L),
$$

which is the explicit form of (9) in this case.

Observe now that the denominator of the last expression is just $\Lambda$ times $U(t ; F)$, while the latter identically equals $u_{F}^{*}$ on $[0, L]$ by the basic equilibrium property. Therefore,

$$
F^{\prime}(t)=\frac{r_{1}}{\Lambda u_{F}^{*}} u(t), \quad t \in(0, L)
$$


We see that $F^{\prime}(t)$ is proportional to $u(t)$ on its support!

It remains to determine $L$ and $u_{F}^{*}$. Recall from Theorem 1 that

$$
u_{F}^{*}=U(L ; F)=r_{1} \int_{L}^{T} u(s) d s .
$$

On the other hand, by (17) and the definition of $L$,

$$
1=F(L)=\int_{0}^{L} F^{\prime}(s) d s=\frac{r_{1}}{\Lambda u_{F}^{*}} \int_{0}^{L} u(s) d s,
$$

which implies

$$
u_{F}^{*}=\frac{r_{1}}{\Lambda} \int_{0}^{L} u(s) d s .
$$

Comparing the last two expressions for $u_{F}^{*}$, we arrive at the following equation, from which $L$ (and hence $u_{F}^{*}$ ) can be computed:

$$
\int_{L}^{T} u(s) d s=\frac{1}{\Lambda} \int_{0}^{L} u(s) d s
$$

Observe that this equation has a unique solution, since the left-hand side in continuously and strictly decreasing to zero, while the right-hand side is continuously increasing from zero.

We summarize these finding in the following Proposition:

Proposition 2 (Equilibrium Profile). For the case of Poisson demand with mean $\Lambda$ and a single board position $(K=1)$, the equilibrium profile $F$ is given in terms of its density $F^{\prime}$ by

$$
F^{\prime}(t)=\frac{1}{\int_{0}^{L} u(s) d s} u(t), \quad t \in(0, L),
$$

where $L$ is the unique solution in $(0, T)$ of equation (20).

For a simple example, consider the case of time-invariant exposure rate, $u(t) \equiv u_{0}$. Here we obtain that the equilibrium profile corresponds to a uniform distribution on $[0, L]$, and from $(20)$ we obtain $(T-L)=L / \Lambda$, so that $L=\frac{\Lambda}{\Lambda+1} T$. Note that for $\Lambda \rightarrow \infty$ (heavy load), $L \rightarrow T$, i.e., the support of the equilibrium profile extends to the entire interval $[0, T]$, as may be expected. This in fact holds for any exposure function $u$, as may be seen from (20).

It may be seen from (21) that the equilibrium density $F^{\prime}(t)$ is proportional to the exposure function $u(t)$. This appears intuitively appealing - higher arrival probabilities are assigned to times with larger utility. However, it is actually remarkable that the arrival rate at time $t$ depends on $u$ only through $u(t)$ at $t$, and not at later times. Indeed, this myopic appearance of the equilibrium is unique to the case of Poisson-distributed demand, as the following converse shows. 
Proposition 3. For the model with $K=1$, suppose that the equilibrium profile is of the form

$$
F^{\prime}(t)=C_{L}^{-1} u(t), \quad t \in[0, L]
$$

for some and $L$, with $F(L)=1$ (here $C_{L}=\int_{0}^{L} u(s) d s$ is the normalization constat). Then the demand $D$ has a Poisson distribution.

Proof. For $K=1$, the utility function (4) reduces to

$$
U(t ; F)=r_{1} \sum_{n \geq 0} p_{D}(n) \int_{t}^{T} B_{0, n}(F(s)-F(t)) u(s) s .
$$

By Theorem $1, U(t ; F)=u^{*}$ on $t \in[0, L]$. In the following let us abbreviate for convenience $F(t)$ as $F_{t}$, and similarly $F^{\prime}(t)$ as $F_{t}^{\prime}$ and $u(t)$ as $u_{t}$. Note that $B_{0, n}(q)=(1-q)^{n}$. Substituting $u_{s}=C_{L} F_{s}^{\prime}$ for $s \leq L$ and $F_{s}=1$ for $s \geq L$, we obtain for each of the last integrals

$$
\begin{aligned}
\int_{t}^{T} B_{0, n}\left(F_{s}-F_{t}\right) u_{s} d s & =\int_{t}^{L}\left(1-F_{s}+F_{t}\right)^{n} C_{L} F_{s}^{\prime} d s+F_{t}^{n} \int_{L}^{T} u_{s} d s \\
& =C_{L}\left[-\frac{1}{n+1}\left(1-F_{s}+F_{t}\right)^{n+1}\right]_{t}^{L}+U_{L} F_{t}^{n} \\
& =\frac{C_{L}}{n+1}\left(1-F_{t}^{n+1}\right)+U_{L} F_{t}^{n},
\end{aligned}
$$

where $U_{L} \triangleq \int_{L}^{T} u_{s} d s$. Substituting the above expressions in the equilibrium equation $U(t ; F)=u^{*}$ gives, for $t \in[0, L]$,

$$
C_{L} \sum_{n \geq 0} \frac{p_{D}(n)}{n+1}\left(1-F_{t}^{n+1}\right)+U_{L} \sum_{n \geq 0} p_{D}(n) F_{t}^{n}=\frac{u^{*}}{r_{1}} .
$$

Observe that $F_{t}$ is continuous and therefore assumes all values in $[0,1]$ as $t$ ranges from 0 to $L$. Therefore, for the last equation to hold for all $t \in[0, L]$, the respective coefficients of each power of $F_{t}$ must sum up to zero. This gives:

$$
-C_{L} \frac{p_{D}(n)}{n+1}+U_{L} p_{D}(n+1)=0, \quad n \geq 0,
$$

or $p_{D}(n+1)=\frac{\Lambda}{n+1} p_{D}(n)$, with $\Lambda=\frac{C_{L}}{U_{L}}$. This implies of course that $p_{D}$ is a Poisson distribution with parameter $\Lambda$.

We turn to consider the efficiency of the Nash equilibrium in this case, which can be computed in closes form.

Proposition 4 (Efficiency). The equilibrium utility is given by

$$
u_{F}^{*}=\frac{1}{1+\Lambda} r_{1} \int_{0}^{T} u(s) d s .
$$

Consequently, the relative efficiency at equilibrium, as defined in (13), equals

$$
\rho(F)=\frac{1}{\left(1+\Lambda^{-1}\right)\left(1-e^{-\Lambda}\right)} .
$$


Proof. The expression for the equilibrium utility follows from (18)-(19), which imply that

$$
\int_{0}^{T} u(s) d s=\frac{\Lambda u_{F}^{*}}{r_{1}}+\frac{u_{F}^{*}}{r_{1}}=\frac{1+\Lambda}{r_{1}} u_{F}^{*} .
$$

Noting that $E\left(D_{0}\right)=E(D)=\Lambda$ under the Poisson distribution, we obtain $S(F)=\Lambda u_{F}^{*}$. Next, evaluating (12) with $K=1$ and $\sum_{n \geq 1}=1-p_{0}(n)=1-e^{-\Lambda}$, we obtain

$$
S^{*}=\left(1-e^{-\Lambda}\right) r_{1} \int_{0}^{T} u(s) d s .
$$

Substituting $S(F)$ and $S^{*}$ in (13) gives the required ratio $\rho(F)$.

Some observations are in order regarding last result.

- It is remarkable that the relative efficiency $\rho(F)$ does not depend on the time-dependent exposure function $u(t)$, but only on the mean demand $\Lambda$.

- The relative efficiency $\rho(F)$, which is smaller than 1 by definition, converges to 1 both when $\Lambda \rightarrow 0$ (where essentially at most one player shares the system) and, more importantly, when $\Lambda \rightarrow \infty$ (heavy demand).

\subsection{Infinite Board with Geometric Utilities}

The results of the previous Subsection can be readily be extended to the asymptotic model of an infinite board, $K=\infty$, with geometrically decreasing relative utility parameters: $r_{k}=r_{1} a^{k-1}, k \geq 1$, with $0<a<1$.

Proceeding formally, with these definitions the expected utility function in (14) becomes,

$$
\begin{aligned}
U(t ; F) & =r_{1} \int_{t}^{T} \sum_{k=0}^{\infty} a^{k} \frac{1}{k !} \Lambda^{k}(F(s)-F(t))^{k} e^{-\Lambda(F(s)-F(t))} u(s) d s \\
& =r_{1} \int_{t}^{T} e^{-\Lambda(1-a)(F(s)-F(t))} u(s) d s .
\end{aligned}
$$

It may be seen that (22) is identical to (15), once $\Lambda(1-a)$ is substituted for $\Lambda$. Thus, all the results of the previous subsection regarding the equilibrium profile $F$ and equilibrium utility $u_{F}^{*}$ are valid in the present model as well, after this substitution.

The relative efficiency can also be evaluated using a similar substitution. To see this, observe that

$$
S(F)=\Lambda u_{F}^{*}=\frac{\Lambda}{1+\Lambda(1-a)} r_{1} \int_{0}^{T} u(s) d s .
$$

Also, evaluating (12) can be seen to give in this case

$$
S^{*}=\frac{1-e^{-\Lambda(1-a)}}{1-a} r_{1} \int_{0}^{T} u(s) d s .
$$


Therefore,

$$
\rho(F)=\frac{S(F)}{S^{*}}=\frac{1}{\left(1+x^{-1}\right)\left(1-e^{-x}\right)}, \quad x \triangleq \Lambda(1-a)
$$

\section{Special Case II: Two Players}

The Poisson demand model of the previous section is well suited for a system with a large number of potential users. In this section we consider the opposite case - where exactly two players compete for position on the advertising board. As in the Poisson case, it will be possible here to attain explicit expressions for the equilibrium profile $F$.

We thus consider two players, where each participates in the game (by submitting an item) with probability $q \in(0,1]$. The exogenous demand $D_{0}$ has the binomial distribution $\operatorname{Bin}(n, p)$ with $n=2$. Accordingly, the endogenous demand $D$ as seen by a participating player is a Bernoulli variate, with $p_{D}(1)=q$ and $p_{D}(0)=1-q$.

Since there are at most two participating players, it is clear that the maximal board size that need be considered is $K=2$. Recall that $r_{1}$ and $r_{2}$ are the relative utility parameters of these two board positions, with $r_{2} \leq r_{1}$. Note that when $r_{1}=r_{2}$ the game becomes trivial, since then the two players are then indifferent between the two board positions. We therefore assume in the following that $r_{2}<r_{1}$. Note further that $r_{2}=0$ corresponds to the single position case, $K=1$. We shall return to this special case as an example towards the end of the section.

With $D \sim \operatorname{Bern}(q)$, the function $g(t, F)$ in (8) becomes

$$
g(t ; F)=q\left(r_{1}-r_{2}\right) \int_{t}^{T} u(s) d s .
$$

Therefore, by Theorem 1,

$$
F^{\prime}(t)=\frac{r_{1} u(t)}{q\left(r_{1}-r_{2}\right) \int_{t}^{T} u(s) d s}=C^{-1} \frac{u(t)}{\int_{t}^{T} u(s) d s}, \quad t \in(0, L),
$$

where $C \triangleq q\left(1-\frac{r_{2}}{r_{1}}\right)<1$. Integrating $F^{\prime}(t)$, we further obtain that for $t \leq L$,

$$
\begin{aligned}
F(t) & =\int_{0}^{t} F^{\prime}(\tau) d \tau=-\left.C^{-1} \ln \left(\int_{\tau}^{T} u(s) d s\right)\right|_{\tau=0} ^{t} \\
& =C^{-1} \ln \left(\frac{\int_{0}^{T} u(s) d s}{\int_{t}^{T} u(s) d s}\right) .
\end{aligned}
$$

It only remains to specify $L$. By the normalization condition $F(L)=1$, we obtain

$$
\int_{L}^{T} u(s) d s=e^{-C} \int_{0}^{T} u(s) d s
$$


and $L$ is the unique solution of this equation. Uniqueness follows by monotonicity in $L$ of the left-hand side. Hence, the equilibrium is unique in this case.

We can now compute the individual and social utility at equilibrium. First, by Theorem 1 and the last equation,

$$
u_{F}^{*}=r_{1} \int_{L}^{T} u(s) d s=r_{1} e^{-C} \int_{0}^{T} u(s) d s .
$$

The social utility at equilibrium is therefore

$$
S(F)=E\left(D_{0}\right) u_{F}^{*}=2 q r_{1} e^{-C} \int_{0}^{T} u(s) d s .
$$

The optimal social utility, as defined in (12), evaluates here as

$$
S^{*}=\left[2 q(1-q) r_{1}+q^{2}\left(r_{1}+r_{2}\right)\right] \int_{0}^{T} u(s) d s .
$$

Therefore,

$$
\begin{aligned}
\rho(F) & =\frac{S(F)}{S^{*}}=\frac{2 q r_{1} e^{-C}}{\left(2 q-q^{2}\right)+q^{2} r_{2}} \\
& =\frac{2 e^{-C}}{2-C} .
\end{aligned}
$$

It is easy to verify that $\rho(F)$ is a strictly decreasing function of the game parameter $C$.

We summarize our findings next.

Proposition 5. For the two-player game with participation probability q:

(i) The equilibrium profile is given by (24), where $C=q\left(1-\frac{r_{2}}{r_{1}}\right)$, and $L$ is the unique solution of equation (25). The equilibrium utility $u_{F}^{*}$ is specified in (26).

(ii) The relative efficiency is given by $\rho(F)=2 e^{-C} /(2-C)$. It approaches 1 as $C \rightarrow 0$, and attains its minimal value of $\rho(F)=\frac{2}{e}$ for $C=1$.

\section{Remarks:}

- Comparing (24) with (21), we see that here $F^{\prime}(t)$ at $t$ does depend on the entire exposure function beyond $t$. In fact, the denominator can be seen to be decreasing in $t$, thereby skewing the arrival density $F^{\prime}(t)$ towards larger arrival times.

- The relative efficiency $\rho(F)$ depends solely on the single parameter $C=$ $q\left(1-\frac{r_{2}}{r_{1}}\right)$. Notably, it does not depend on the exposure function $u(t)$.

- The extreme case of $C \rightarrow 0$ corresponds either a small joining probability $q \rightarrow 0$ for each player, or to $r_{2} \approx r_{1}$. These values minimize the conflict between the players, which explains the convergence of the relative efficiency to 1 . Indeed, it may be seen from (25) that $L \rightarrow 0$ correspondingly, and the equilibrium profile $F$ approaches a unit atom at $t=0$. 
- The case of $C=1$ corresponds to a deterministic number of participants $\left(D_{0}=2\right)$, and a single board position (as $\left.r_{2}=1\right)$. As observed, the relative efficiency in this case equals $2 / e$, independently of the exposure function $u(t)$.

Example. We close the section with the simple example of constant exposure rate: Suppose $u(t)=u_{0}, t \in[0, T]$. From (25) we obtain that $(T-L)=e^{-C} T$, or

$$
L=\left(1-e^{-C}\right) T
$$

and by (24),

$$
F^{\prime}(t)=\frac{C^{-1}}{T-t}, \quad t \in(0, L)
$$

We observe that the density $F^{\prime}$ is strictly increasing in $t$, from $F^{\prime}(0)=$ $(T C)^{-1}$ to $F^{\prime}(L)=\frac{C^{-1}}{T-L}=e^{C}(T C)^{-1}$.

\section{$6 \quad$ Numeric Examples}

We present next some numerical computations, that illustrate the equilibrium profile obtained for different choices of model parameters. We also compare the numerical results to our theoretical findings. The numerical results are obtained using the computational procedures outlined in Section 6, implemented in MAT$\mathrm{LAB}$. The basic Euler method is used to integrate the relevant differential equations.

We present two case studies - one with deterministic demand, and the other with Poisson demand. In both cases we assume a uniform exposure function, $u(t)=1$ for $t \in[0, T]$. In view of Remark 1 in Section 6 , the obtained equilibria for other exposure functions will be similar up to a time change. We further fix the time horizon at $T=10$.

Case 1: Deterministic demand, with single board position $(B=1)$.

We first consider a deterministic number of players, and a single board position. The obtained equilibria profiles are shown in Figure 1 for $N=2,4,11,51$ players (note that the total number of players is $D+1$ ). We note that for $B=1$ the equilibrium is unique by Theorem 2. For $N=2$, the numeric results coincide with the theoretical results of Subsection 5. Indeed, following the example at the end of that subsection with $C=1$, we obtain $F^{\prime}(t)=1 /(T-t)$, and $L=\left(1-e^{-1}\right) T=6.3$.

As the demand increases, $L$ is seen to approach $T$, and the equilibrium distribution is close to uniform. This can be explained with the help of a high-demand fluid model, which is outside the scope of the present paper.

Case 2: Poisson demand.

Here we consider the system with a fixed Poisson demand, $D, D_{0} \sim \operatorname{Poiss}(\Lambda)$, with $\Lambda=3{ }^{\star \star}$ Several values for the board size $K$ were considered, with relative

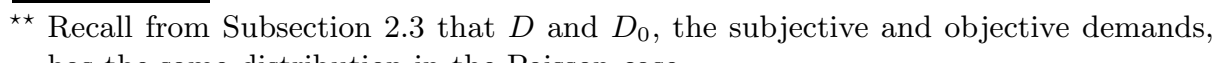
has the same distribution in the Poisson case. 


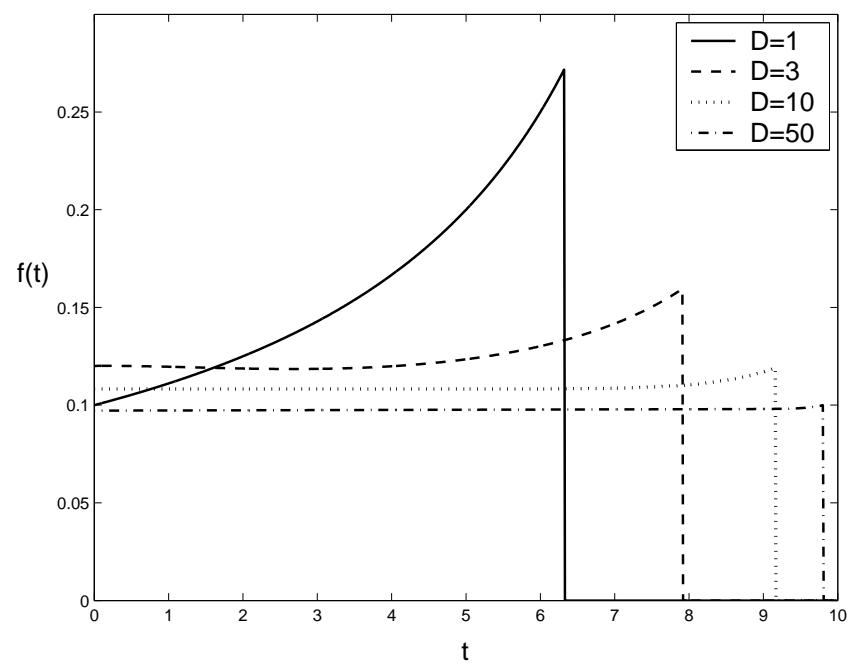

Fig. 1: The equilibrium density functions for deterministic demand (Case 1). The number of players is $N=D+1$.

utility parameters than decrease geometrically according to $r_{k}=(0.5)^{k-1}, k=$ $1, \ldots, K$. Thus for $K=1$ we have $r_{1}=1$, and for $K=3$ we have $\left(r_{1}, r_{2}, r_{3}\right)=$ $\left(1, \frac{1}{2}, \frac{1}{4}\right)$.

The results obtained for $K=1,2,3,6$ are depicted in Figure 2 . For $K=1$, the results confirm the theoretical prediction in Proposition 2, namely a uniformlydistributed equilibrium profile with $L$ solving equation (20). Since $u=1$ this equation gives $(10-L)=L / 3$, or $L=7.5$.

For $K=6$, since the $r_{k}$ 's decay exponentially, the model can be approximated by the asymptotic model with $K=\infty$ that was studied in Subsection 4.2. As observed there, the obtained equilibrium is identical to the one obtained for a single-position case, with an effective demand of $\Lambda^{\prime}=\Lambda(1-a)=1.5$. Thus, we expect a uniform distribution on $[0, L]$, with $L$ determined from $(10-L)=L / 1.5$, which gives $L=6$. This is indeed what was obtained numerically.

In contrast to the above extreme case, for for intermediate values of $K=2$ and $K=3$, the equilibrium distribution may be seen to be non-uniform.

\section{Conclusion}

We have considered in this a stylized model of advertiser timing competition, where advertisers (or other media users) compete for position over a shared publication medium that gives priority to recently submitted items. Our results address the existence, uniqueness, and computation of the symmetric equilibrium profile, as well as some explicit solutions for certain special cases of the model.

The basic model of this paper may be enriched in several directions, that include: 


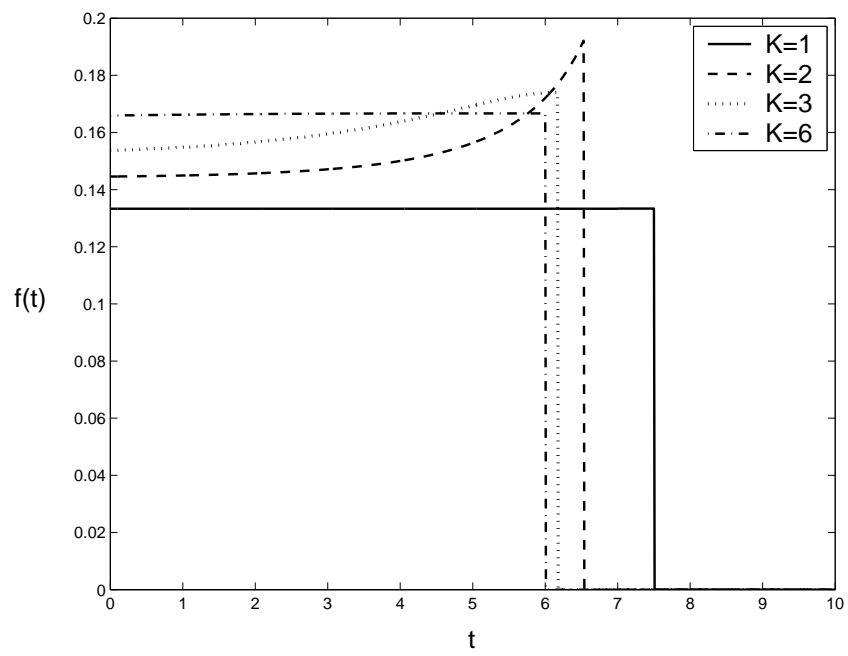

Fig. 2: The equilibrium density functions for Poisson demand (Case 2).

- Additional cost components, such as a one-time submission cost.

- Long-rage periodic model: Our model assumes a finite and per-specified display interval $[0, T]$. It is of practical interest to consider a variant of this model that operates on a long (indefinite) duration, with a continuous supply of new ads (which may fluctuate periodically, say on a daily basis, along with the exposure function $u(t))$.

- Large-demand limits: When the number of submitted ads is large, fluid-scale models that assumes a deterministic rather than stochastic submission rates may be computationally attractive. The analysis of such fluid models and their relations to the stochastic ones are of interest here.

- Multi-class models: It may be of interest to consider several classes of advertisers with different preferences - for example, the exposure function $u(t)$ may differ among advertisers that address different segments of the population.

These topics present challenges with various degrees of difficulty, which are left for future investigation.

\section{References}

1. J. Constine, "Minimizing Your Facebook Ad CPC Bid Prices by Avoiding Bid Competition," http://www.insidefacebook.com/2011/09/08/suggested-bid-cpc/, Sept. 2011.

2. R. B. Cooper, Introduction to Queueing Theory, 2nd Ed., North-Holland, New York, 1981.

3. L. Einav, "Seasonality in the U.S. Motion Picture Industry," RAND Journal of Economics 38(1):127-45, 2007. 
4. D. Fudenberg and J. Tirole, Game Theory, MIT press, Cambridge, MA, 1991.

5. A. Glazer and R. Hassin, "?/M/1: On the equilibrum distribution of customer arrivals," European J. of Oper. Research 13:146-150, 1983.

6. J. K. Hale and S. M. Verduyn Lune, Introduction to Functional Differential Equations, Springer, New York, 1993.

7. R. Hassin and Y. Kleiner, "Equilibrium and optimal arrival patterns to a server with opening and closing times," IEE Transactions 43(3):164-175, March 2011.

8. M. Haviv, "When to arrive at a queue with tardiness costs?" Performance evaluation 70:387-399, 2013.

9. R. Jain, S. Juneja and N. Shimkin, "The Concert Queueing Game: To Wait or to be Late," Discrete Event Dynamic Systems 21(1):103-138, April 2011.

10. S. Juneja and N. Shimkin, "The Concert Queueing Game: Strategic arrivals with waiting and tardiness costs," Queueing Systems 74(4):369-402, August 2013.

11. T. Klastorin and W. Tsai, "New Product Introduction: Timing, Design, and Pricing," Manufacturing and Service Operations Management 6(4):302-320, 2004.

12. R. E. Krider and C. B. Weinberg, "Competitive Dynamics and the Introduction of New Products: The Motion Picture Timing Game," Journal of Marketing 35(1):115, 1998.

13. R. Laraki, E. Solan and N. Vieille, "Continuous-time Games of Timing," Journal of Economic Theory 120(2):206-238, February 2005.

14. R. Lindley, "Existence, uniqueness, and trip cost function properties of user equilibrium in the bottleneck model with multiple user classes," Transportation Science 38(3):293-314, 2004.

15. Z. Lotker, B. Patt-Shamir, and M. R. Tuttle, "A game of timing and visibility," Games and Economic Behavior 62(2):643-660, 2008.

16. S. Radas and S. M. Shugan, "Seasonal Marketing and Timing New Product Introductions," Journal of Marketing Research 35(3):296-315, 1998.

17. D. Rajagopal, "New Product Introduction and Seasonality Effect in Food Products Retailing," Journal of Food Products Marketing 14(4):54-75, 2008.

18. W. S. Vickrey, "Congestion theory and transport investment," The American Economic Review 59:251-260, 1969. 


\section{Appendix}

\section{A Proofs for Section 3}

We next present the proofs for our main results in the previous Section, namely Theorems 1-2 and Proposition 1. We start with the latter, which is also needed for the proofs of the two other theorems.

Proof of Proposition 1: Fixing $L$, we consider the differential equation (11) with the stated terminal conditions over $t \in\left[t_{L}, L\right]$. Denote the right hand side of (11) by $H(t, F) \triangleq r_{1} u(t) / g(t, F)$. Since $H$ is non-negative it follows that $F^{\prime} \geq 0$, and noting that $F_{L}(L)=1$ we may restrict attention to non-decreasing functions $F$ with values in $[0,1]$. For simplicity, when $t_{L}>0$ we may set $F_{L}(t)=0$ for $t<t_{L}$. We further consider $F_{L}$ to be a member of the Banach space of continuous functions on $[0, T]$, equipped with the sup-norm topology.

(i) As mentioned, Equation (11) is a retarded FDE in the sense of [6]. Existence and uniqueness of solutions follow by standard results for these equations, once we verify that the right hand side has appropriate continuity and boundedness properties. Observing $H$ above, recall that $u(t)$ is a continuous and bounded function. It is easily verified by straightforward bounds that $g$ in (8) is (Lipschitz) continuous in $t$ and in $F$ (with the sup-norm topology). Therefore, $H(t, F)$ is also Lipschitz continuous provided that $g(t, F)$ is bounded away from zero.

We first show that the latter property of $g$ holds for any $\epsilon>0$ over $t \epsilon$ $\left[t_{L}(\epsilon), L\right]$, where

$$
t_{L}(\epsilon)=\inf \{s \in[0, L): F(s)>\epsilon\} .
$$

Indeed, for such $t$, we obtain from (8) that

$$
g(t, F) \geq\left(r_{1}-r_{2}\right) \sum_{n \geq 0} q(n) \int_{L}^{T} B_{0, n}(F(s)-F(t)) u(s) d s
$$

where $q(n) \triangleq(n+1) p_{D}(n+1)>0$. Observe that $r_{1}-r_{2}>0$ by assumption, and similarly $q\left(n_{0}\right)>0$ for some $n_{0} \geq 1$, since $E(D)>0$ by assumption. Furthermore, $F(s)=1$ for $t \geq L, F(t) \geq \epsilon$ for $t \in\left[t_{L}(\epsilon), L\right]$, and $B_{0, n}(p)=$ $(1-p)^{n}$ is decreasing in $p$. Therefore,

$$
\begin{aligned}
g(t, F) & \geq\left(r_{1}-r_{2}\right) q\left(n_{0}\right) u_{\min } \int_{L}^{T} B_{0, n}(1-\epsilon) d s \\
& =\left(r_{1}-r_{2}\right) q\left(n_{0}\right) u_{\min } B_{0, n_{0}}(1-\epsilon)(T-L) \triangleq \zeta(\epsilon, L)>0 .
\end{aligned}
$$

If follows that $H(t, F)$ is indeed continuous in $(t, F)$ for $t \in\left[t_{L}(\epsilon), L\right]$. Existence of a unique local solution to $F^{\prime}(t)=H(t, F)$ for $t \leq L$ now follows by Theorem 2.1 in [6]. Since $H(t, F) \leq r_{1} u_{\max } / \zeta(\epsilon, L)<\infty$, it follows by Theorem 3.1 there that the solution $F(t)$ can be extended to $t \in\left[t_{L}(\epsilon), L\right]$. Finally, by taking $\epsilon \rightarrow 0$, so that $t_{L}(\epsilon) \rightarrow t_{L}$, it follow that a unique solution $F$ exists on $\left[t_{L}, L\right]$. 
(ii) We wish to show that $g\left(t, F_{L}\right)$ is bounded away from 0 over $t \in\left[t_{L}, L\right]$ . It was already shown in $(i)$ that $g(t, F) \geq \zeta(\epsilon, L)>0$ for $t \in\left[t_{L}(\epsilon), L\right]$ and any $\epsilon>0$. However, since $\zeta(\epsilon, L) \rightarrow 0$ as $\epsilon \rightarrow 0$, we need to strengthen this bound for the solution $F_{L}$. Setting $\epsilon=0.5$, we have from that bound that $g\left(t, F_{L}\right) \geq \zeta(0.5, L) \triangleq \delta_{L}>0$ for $t \in\left[t_{L}(0.5), L\right]$. Clearly, if $t_{L}(0.5)=0$ we are done. Otherwise, we still need to bound $g(t, F)$ for $t<t_{L}(0.5)$. Since $F_{L}$ is a continuous function, there exists an interval $I_{L} \subset\left[t_{L}(0.5), L\right]$ of length $\left|I_{L}\right|>0$, over which $F_{L}(t) \in[0.5,0.6]$. (In fact, since $g(t, F) \geq \delta_{L}$ implies that $F^{\prime}(t) \leq r_{1} u_{\max } / \delta_{L}$, it may be seen that $\left|I_{L}\right|>0.1 \delta_{L} / r_{1} u_{\max }$.) Therefore, similarly to $(27)$, we obtain that

$$
\begin{aligned}
g\left(t, F_{L}\right) & \geq\left(r_{1}-r_{2}\right) q\left(n_{0}\right) u_{\min } \int_{s \in I_{L}} B_{0, n}\left(F_{L}(s)-F_{L}(t)\right) d s \\
& \geq\left(r_{1}-r_{2}\right) q\left(n_{0}\right) u_{\min }\left|I_{L}\right| B_{0, n}(0.6), \quad t \in\left[t_{L}, t_{L}(0.5)\right],
\end{aligned}
$$

where we have used that fact that $F_{L}(s) \leq 0.6$ for $s \in I_{L}$, so that $F_{L}(s)-F_{L}(t) \leq$ 0.6 as well. Using the above-mentioned bound on $\left|I_{L}\right|$, we finally obtain

$$
g\left(t, F_{L}\right) \geq 0.1 \frac{r_{1}-r_{2}}{r_{1}} q\left(n_{0}\right) \frac{u_{\min }}{u_{\max }} \delta_{L} B_{0, n}(0.6) \triangleq \eta_{L}>0, \quad t \in\left[t_{L}, t_{L}(0.5)\right] .
$$

This provides the required uniform bound over $g\left(t, F_{L}\right)$ for $t<t_{L}(0.5)$. Combining the above bounds on $g$, we obtain that $g\left(t, F_{L}\right) \geq \min \left\{\delta_{L}, \eta_{L}\right\} \triangleq \epsilon_{L}>0$ for $t \in\left[t_{L}, L\right]$, as stated.

(iii) Continuity in $L$ of the solutions $F_{L}$ to our equation $F_{L}^{\prime}(t)=H\left(t, F_{L}\right)$ follows by Theorem 2.2 (Continuous dependence) in [6], once we show each $L \in(0, T)$ has a neighborhood $N_{L}=(L-\epsilon, L+\epsilon)$ such that $H\left(t, F_{L^{\prime}}\right)$ is uniformly bounded over all $t$ and solutions $F_{L^{\prime}}, L^{\prime} \in N_{\epsilon}(L)$. Since $H(t, F) \leq$ $r_{1} u_{\max } / g(t, F)$, the required uniform boundedness follows by observing the bound $g\left(t, F_{L}\right) \geq \epsilon_{L}>0$ developed in item (ii) above, and further noting that $\epsilon_{L}$ is continuous (in fact linear) in $L$, as may be seen in the above proof. Continuity of $t_{L}$ in $L$ follows immediately from that of the solution $F_{L}$.

This completes the proof of Proposition 1.

We next address the proof of Theorem 1, which relies on several Lemmas. The next two establish properties $(i),(i i)$ and $(i v)$ of the Theorem.

For a given distribution function $F$, let $\eta_{F}$ denote the corresponding probability measure on the reals. Recall that $\operatorname{supp}(F)$ denotes the support of $\eta_{F}$, i.e., the smallest closed set of $\eta_{F}$-probability 1 .

Lemma 1. Let $F$ be an equilibrium profile. Then

(i) $F(t)$ is a continuous function of $t \in[0, T]$ (i.e, $\eta_{F}$ does not contain any point masses).

(ii) $U(t, F)$ is a continuous function of $t \in[0, T]$.

(iii) $\operatorname{supp}(F)$ is an interval $[0, L]$, with $L<T$.

Proof. (i) Suppose $\eta_{F}$ contains a point mass at $t$, namely $F(t)-F(t-)=q>$ 0 . We claim that, for $\epsilon>0$ small enough, $U(t+\epsilon ; F)$ will be strictly larger 
than $U(t ; F)$. But this contradicts the equilibrium properties in (5)-(6), since $U(t, F)<u^{*} \triangleq \max _{s} U(s, F)$ and $\eta_{F}(\{t\})>0$.

To see that $U(t ; F)<U(t+\epsilon ; F)$ for $\epsilon$ small enough, consider a specific player $i$ who arrives at $t$, and note that having a point mass at $t$ implies that, with positive probability, at least one other player arrives at $t$ simultaneously. Recall that the ordering of simultaneous arrivals is random, so that in that case player $i$ has a probability of at least 0.5 to be immediately placed in position 2 or worse. On the other hand, arriving at a slightly later time $t+\epsilon$ guarantees that the player will be placed higher than all other arrivals at $t$, thereby strictly improving his expected utility over $[t+\epsilon, T]$ relative to the previous case by a quantity $\delta$ which is bounded away from 0 even as $\epsilon \rightarrow 0$, while the utility loss over $[t, t+\epsilon]$ is bounded by $\epsilon r_{1}^{*}$, which is arbitrarily small. Here $r_{1}^{*} \triangleq r_{1} \max _{t \in[0, T]} u(t)$.

(ii) Again, we compare $U(t ; F)$ to $U(t+\epsilon ; F)$. For $\epsilon>0$, since there are no point masses in $\eta_{F}$ (as just established), then $\eta_{F}\{[t, t+\epsilon]\} \rightarrow 0$ as $\epsilon \rightarrow 0$, so that the probability $\delta(\epsilon)$ of an arrival on that interval converges to 0 as $\epsilon \rightarrow 0$. Now, a player that arrives at $t+\epsilon$ rather than $t$ will incur a utility loss of at most $\epsilon r_{1}^{*}$ over $[t, t+\epsilon]$, and a utility gain of $\delta(\epsilon) T r_{1}^{*}$ at most over $[t+\epsilon, T]$. Therefore, $\lim _{\epsilon \rightarrow 0}|U(t+\epsilon ; F)-U(t, F)| \leq r_{1}^{*}|\epsilon-\delta(\epsilon) T|$ converges to 0 as $\epsilon \rightarrow 0$, establishing continuity from the right. A similar argument holds for $\epsilon<0$.

(iii) To show that the $\operatorname{support} \operatorname{supp}(F)$ is an interval of the form $[0, L]$, suppose to the contrary that there exists an interval $(t, s), t<s$ such that $\eta_{F}\{[t, s]\}=0$ and $F(s)<1$. We can extend $s$ to the right while keeping the above properties till it hits $\operatorname{supp}(F)$. Then, by property (5) of the NEP and the above-established continuity of $U(\cdot ; F)$, it follows that $U(s ; F)=u^{*}$. But now, since there are no arrivals on $(t, s)$, an arrival at $s-\epsilon$ will remain in position 1 until time $s$, implying that $U(s-\epsilon ; F) \geq U(s ; F)+\epsilon r_{1}\left[\min _{t \in[s-\epsilon, s]} u(t)\right]>u^{*}$, which contradicts the definition of $u^{*}$.

Finally, we show that $L<T$. It is clear that $U(T-\epsilon ; F) \leq \epsilon r_{1}^{*}$. Therefore, $U(t ; F)<u^{*}$ for $t>t_{0}=T-u^{*} / r_{1}^{*}$, implying that $\left(t_{0}, T\right]$ does not belong to $\operatorname{supp}(F)$.

Lemma 2. Any equilibrium profile $F$ satisfies properties $(i),(i i),($ iv $)$ of Theorem 1 .

Proof. Property $(i)$ was established in Lemma 1. For property $(i i)$, suppose $F$ is an equilibrium profile. Since $U(\cdot ; F)$ is continuous by Lemma $1(i i)$, it follows by property $(i)$ and the definition of the equilibrium that $U(t ; F)=u_{F}^{*}$ on $[0, L]$, which is property $(i i)$. Conversely, suppose that some continuous strategy $F$ satisfies property $($ ii $)$. By definition of the equilibrium, to establish that $F$ is indeed an equilibrium strategy it only remains to show that $U(t ; F) \leq u_{L}$ for $t>L$. But since there are no other arrivals on $[L, T]$ under $F$, then any arrival there would remain in the first position till $T$, so that $U(t ; F)=r_{1} \int_{t}^{T} r_{1} u(s) d s$ for $t \in[L, T]$. Since $u(s)>0$ it follows that $U(t ; F)<U(L ; F)=u_{L}$ for $t>L$, as required, and property $(i i)$ is established. Property $(i v)$ follows by observing that $u_{L}^{*}=U(L ; F)$ and substituting the last integral. 
It remains to establish the differential characterization of Theorem $1(\mathrm{iii})$, and the existence claim in that Theorem. We begin by computing the time derivative of the utility function $U(t ; F)$ in $(4)$.

Lemma 3. For any strategy $F$, the following hold:

(i) At any point $t$ at which $F$ is differentiable,

$$
\frac{d}{d t} U(t ; F)=-r_{1} u(t)+F^{\prime}(t) g(t, F),
$$

where $g$ is defined in equation (8).

(ii) Conversely, if $U$ is differentiable at $t$ and $g(t, F)>0$ there, then $F$ is differentiable at $t$, hence $F^{\prime}(t)$ satisfies Equation (28).

(iii) The function $g(\cdot ; F)$ is continuous over $t \in[0, T]$, and strictly positive for all $t<T$ (except for the degenerate case where $F\left(T^{-}\right)=0$, in which $g \equiv 0$ ).

Proof. (i) By formally differentiating (4) under the integral sign,

$$
\begin{aligned}
\frac{d}{d t} U(t ; F) & =-\left.\sum_{k=0}^{K-1} r_{k+1} \mathbb{P}_{F}\left(N_{(t, s]}=k\right) u(s)\right|_{s=t}+\int_{t}^{T} \sum_{k=0}^{K-1} r_{k+1} \frac{\partial}{\partial t} \mathbb{P}_{F}\left(N_{(t, s]}=k\right) u(s) d s \\
& =-r_{1} u(t)+\int_{t}^{T} \sum_{k=0}^{K-1} r_{k+1} \frac{\partial}{\partial t} \mathbb{P}_{F}\left(N_{(t, s]}=k\right) u(s) d s .
\end{aligned}
$$

Further, observing (3),

$$
\begin{aligned}
\frac{\partial}{\partial t} \mathbb{P}_{F}\left(N_{(t, s]}=k\right) & =\sum_{n \geq k} p_{D}(n) \frac{\partial}{\partial t} B_{k, n}(F(s)-F(t)) \\
& =-\left.\sum_{n \geq k} p_{D}(n) F^{\prime}(t) \frac{\partial}{\partial p} B_{k, n}(p)\right|_{p=F(s)-F(t)}
\end{aligned}
$$

Recalling the definition of $B_{k, n}$ in (2),

$$
\begin{aligned}
\frac{\partial}{\partial p} B_{k, n}(p) & =\left(\begin{array}{l}
n \\
k
\end{array}\right) k p^{k-1}(1-p)^{n-k} \mathbf{1}_{\{k>0\}}-\left(\begin{array}{l}
n \\
k
\end{array}\right)(n-k) p^{k}(1-p)^{n-k-1} \mathbf{1}_{\{k<n\}} \\
& =n B_{k-1, n-1}(p)-n B_{k, n-1}(p)
\end{aligned}
$$

(where we use the convention that $B_{-1, n+1}=B_{n, n-1}=0$ ), so that

$$
\begin{aligned}
& \frac{d}{d t} U(t ; F)=-r_{1} u(t)+\ldots \\
& +F^{\prime}(t) \int_{t}^{T} \sum_{k=0}^{K-1} r_{k+1} \sum_{n \geq k} p_{D}(n) n\left[B_{k, n-1}(p)-B_{k-1, n-1}(p)\right]_{p=F(s)-F(t)} u(s) d s .
\end{aligned}
$$


It remains to simplify the sum in the last expression. Denoting $q_{n}=n p_{D}(n)$, observe that

$$
\begin{aligned}
\sum_{k=0}^{K-1} r_{k+1} & \sum_{n \geq k} q_{n}\left[B_{k, n-1}(p)-B_{k-1, n-1}(p)\right] \\
= & \sum_{k=0}^{K-1} r_{k+1} \sum_{n \geq k-1} q_{n+1} B_{k, n}(p)-\sum_{k=-1}^{K-2} r_{k+2} \sum_{n \geq k} q_{n+1} B_{k, n}(p) \\
= & \sum_{k=0}^{K-1} r_{k+1} \sum_{n \geq k} q_{n+1} B_{k, n}(p)-\sum_{k=0}^{K-1} r_{k+2} \sum_{n \geq k} q_{n+1} B_{k, n}(p) \\
= & \sum_{k=0}^{K-1}\left(r_{k+1}-r_{k+2}\right) \sum_{n \geq k} q_{n+1} B_{k, n}(p)
\end{aligned}
$$

where the first equality follows by an index shift, and the second by observing again that $B_{k, n} \equiv 0$ for $n=k-1$ or $k=-1$, while $r_{K+1}=0$ by definition. Substituting (33) in (32) gives the equality in (28), with $g$ as defined in (8).

Existence of the derivative of $U$ at $t$ now follows by observing that, on the right-hand side of (28), both $u$ and $g$ are continuous functions of $t$ (the first by assumption, and the second by its definition as an integral).

(ii) Recall that $u(t)$ is continuous by assumption, and so is $g$ (as observed in $\left(\right.$ iii)). Observing (28) and the assumed positivity of $g(t, F)$, existence of $F^{\prime}(t)$ may now be inferred by basic calculus from the expression (4) for $U$.

(iii) Observing (8), continuity follows by the definition of $g$ as an integral over a bounded function. Note that the sum $\sum_{n \geq k}(n+1) p_{D}(n+1)$ is bounded by $E(D)$, which is finite by assumption. Strict positivity of $g(t, F)$ follows as in Proposition 1(ii).

The next Lemma establishes the differential characterization of the equilibrium in Theorem 1.

Lemma 4. Part (iii) of Theorem 1 holds true. That is,

(i) A continuous distribution function $F:[0, T] \rightarrow[0,1]$ that satisfies the properties in Theorem 1(iii) is an equilibrium profile.

(ii) Conversely, any equilibrium profile $F$ satisfies the properties stated in Theorem $1($ iii).

Proof. ( $i$ ) From (9) and (28) we obtain that $\frac{d}{d t} U(t ; F)=0$ for $t \in(0, L)$. Since $U$ is continuous it follows that $U(t ; F)=u, t \in[0, L]$ for some constant $u$. Therefore, by Theorem $1(i i), F$ is an equilibrium profile.

(ii) Suppose $F$ is an equilibrium profile. In view of Theorem $1(i i)$, it only remains to verify (9). Now, Theorem $1(i i)$ also implies that $U(t ; F)=u_{F}$ for $t \in[0, L]$, hence $\frac{d}{d t} U(t ; F)=0$ for $t \in(0, L)$. Observing Lemma 3(ii)-(iii) and Equation (28), it follows that $-r_{1} u(t)+F^{\prime}(t) g(t, F)=0$ (with $g(t, F)>0$ ), and Equation (9) follows. 
Lemma 5. There exists an equilibrium profile $F$.

Proof. We demonstrate existence of a distribution function $F$ that satisfies the requirements of Theorem $1($ iii $)$. For each $L \in(0, T)$, let $F_{L}(t), t \in\left[t_{L}, T\right]$ denote the solution of Equation $F_{L}(t)$ as defined in Proposition 1. Note that $F_{L}\left(t_{L}\right)=0$ if $t_{L}>0$. To simplicity the exposition, if $t_{L}>0$ extend $F_{L}$ linearly up to $t=0$ using $F_{L}(t)=-\left(t_{L}-t\right), t \in\left[0, t_{L}\right]$. By part (iii) of Proposition $1, F_{L}(0)$ is continuous in $L$. We show below that $t_{L}>0$ (hence $\left.F_{L}(0)<0\right)$ for $L$ close enough to $T$, while $F_{L}(0)>0$ for $L$ close enough to 0 . By the above-mentioned continuity, this implies that $F_{L}(0)=0$ for some intermediate $L \in(0, T)$. It follows that for this value of $L, F_{L}$ satisfies all the requirements in Theorem $1($ iii), and is therefore an equilibrium profile.

To verify that $F_{L}(0)>0$ for $L$ close enough to 0 , observe that $g(t, F)$ in (8) is bounded away from zero while $F(t)$ is positive: In particular, using the bound in Equation (27), if $L \leq T / 2$ and $F(t) \geq 0.5$ we have

$$
g(t, F) \geq \zeta\left(0.5, \frac{1}{2} T\right) \triangleq \zeta_{0}>0,
$$

Therefore $F_{L}^{\prime}(t)=r_{1} u(t) / g\left(t, F_{L}\right) \leq r_{1} u_{\max } / \zeta_{0}$ under these conditions, and since $F_{L}(L)=1$ this implies that $F_{L}(0) \geq 0.5$ if $L<\frac{1}{2} \zeta_{0} / r_{1} u_{\max }$.

To verify that $t_{L}>0$ for $L$ close enough to $T$, observe from (8) that $g$ is upper bounded by

$$
g(t, F) \leq r_{1} E(D) \int_{t}^{T} u(s) d s .
$$

Therefore, by (9),

$$
F_{L}^{\prime}(t)=\frac{r_{1} u(t)}{g\left(t, F_{L}\right)} \geq E(D)^{-1} \frac{u(t)}{\int_{t}^{T} u(s) d s}, \quad t \in\left[t_{L}, L\right] .
$$

By integration, this implies

$$
F_{L}(L)-F_{L}(t) \geq E(D)^{-1} \ln \left(\frac{\int_{t}^{T} u(s) d s}{\int_{L}^{T} u(s) d s}\right) .
$$

Since the last denominator is arbitrarily small for $L$ close enough to $T$, and recalling that $u(s)>0$, it follows that $F_{L}(t)=0$ for some $t>0$ (namely $t_{L}>0$ ) for such $L$.

This completes the proof of existence of an equilibrium profile.

Proof of Theorem 1: Items (i)-(iv) of this Theorem were proven in Lemmas 2 and 4, while existence of an equilibrium profile was established in Lemma 5.

We may now proceed to the proof of the uniqueness claim in Theorem 2. The proof relies on the following monotonicity properties of the function $g$, as defined in (8).

Lemma 6. Let Assumption 2 hold. 
(i) For each $n \geq 0$, the function

$$
f_{n}(p)=\sum_{k=0}^{K-1}\left(r_{k+1}-r_{k+2}\right) B_{k, n}(p), \quad p \in[0,1]
$$

is non-increasing in $p$.

(ii) Consequently, let $F_{1}$ and $F_{2}$ be two strategy profiles (namely distribution functions over $[0, T]$ ) such that, for some $t \in[0, T)$,

$$
F_{2}(s)-F_{2}(t) \geq F_{1}(s)-F_{1}(t) \text { for all } s \in[t, T] .
$$

Then $g\left(t, F_{2}\right) \leq g\left(t, F_{1}\right)$.

(iii) If, in addition, the inequality in (34) is strict over some nonempty interval $I \subset[t, T]$, then either $g\left(t, F_{2}\right)<g\left(t, F_{1}\right)$, or else $g\left(\tau, F_{2}\right)=g\left(\tau, F_{1}\right)$ for all $\tau \in[0, T]$.

Proof. ( $i$ ) Denote $\delta_{k}=r_{k}-r_{k+1}$. A similar calculation to equations (31) and (33) yields,

$$
f_{n}^{\prime}(p) \triangleq \frac{d}{d p} f_{n}(p)=n \sum_{k=1}^{K}\left(\delta_{k+1}-\delta_{k}\right) B_{k-1, n-1}(p)
$$

But Assumption 2 implies that $\delta_{k+1}-\delta_{k}=2 r_{k+1}-r_{k}-r_{k+2} \leq 0$, so that $f_{n}^{\prime}(p) \leq 0$.

(ii) Changing the order of summation in (8), and recalling that $B_{k, n} \equiv 0$ for $k>n, g$ may be rewritten as

$$
g(t, F)=\sum_{n \geq 0} q_{n} \int_{t}^{T} f_{n}(F(s)-F(t)) d s,
$$

where $q_{n}=(n+1) p_{D}(n+1) \geq 0$. The claim now follows directly from $(i)$ and the assumed relation (34) between $F_{1}$ and $F_{2}$.

(iii) For each $n \geq 0$, it may be seen from (35) that either $q_{n} f_{n}^{\prime}(q) \equiv 0$ for $q \in[0,1]$, or $q_{n} f_{n}^{\prime}(q)<0$ for all $q \in(0,1)$. Suppose first that the former holds for all $n \geq 0$. Then, by $(36), g(t, F)$ does not depend on $F$, so that $g\left(\cdot, F_{1}\right)=g\left(\cdot, F_{2}\right)$. Otherwise, suppose that the latter holds for some $m \geq 0$, namely $q_{m} f_{m}^{\prime}(q)<0$ for $q \in(0,1)$. Then, wherever (34) holds with a strict inequality,

$$
q_{m} f_{m}\left(F_{2}(s)-F_{2}(t)\right)<q_{m} f_{m}\left(F_{1}(s)-F_{1}(t)\right),
$$

which holds over $s \in I$. Substituting in (36) obtains $g\left(t, F_{2}\right)<g\left(t, F_{1}\right)$.

Proof of Theorem 2 (uniqueness): Recall that an equilibrium profile $F$ satisfies the properties in Theorem 1 with some parameter $L$. In particular, $F(t)=1$ on $t \in[L, T], F$ satisfies equation $(9)$ on $[0, L]$, and $F(0)=0$. Let $F_{1}$ and $F_{2}$ denote two equilibrium profiles with corresponding parameters $L_{1}$ and $L_{2}$. We will show below that $L_{1}<L_{2}$ implies that $F_{2}(0)<F_{1}(0)$, so that only 
one can be an equilibrium. Therefore $L_{1}=L_{2}$. But Proposition 1 implies that $L$ defines $F$ uniquely, hence the equilibrium is unique.

Consider then $F_{1}$ and $F_{2}$ as above, and suppose that $L_{1}<L_{2}$. Since $F_{1}(s)=1$ for $s \in\left[L_{1}, T\right]$ and $F_{2}$ is strictly increasing over $t<L_{2}$, it follows that

$$
F_{2}\left(L_{1}\right)<F_{1}\left(L_{1}\right)=1,
$$

and

$$
F_{2}(s)-F_{2}\left(L_{1}\right)>F_{1}(s)-F_{1}\left(L_{1}\right)=0, \quad s \in\left(L_{1}, T\right] .
$$

Therefore inequality (34) is satisfied with strict inequality for $t=L_{1}$. By Lemma 6(iii), exactly one of the following two conclusions holds:

(a) $g\left(t, F_{2}\right)=g\left(t, F_{1}\right)$ for all $t \in[0, T]$. In that case it follows from (9) that $F_{2}^{\prime}(t)=F_{1}^{\prime}(t)$ holds for $t \leq L_{1}$, so that

$$
F_{1}(0)-F_{2}(0)=F_{1}\left(L_{1}\right)-F_{2}\left(L_{1}\right)>0 .
$$

(b) $g\left(t, F_{2}\right)<g\left(t, F_{1}\right)$ at $t=L_{1}$. By (9), this implies that $F_{2}^{\prime}\left(L_{1}\right)>F_{1}^{\prime}\left(L_{1}^{-}\right)$. We argue that this inequality extends to all $t<L_{1}$. Suppose, to the contrary, that $F_{2}^{\prime}(\tau) \leq F_{1}^{\prime}(\tau)$ for some $\tau<L_{1}$. Noting that $F_{1}^{\prime}$ and $F_{2}^{\prime}$ are continuous on $\left[0, L_{1}\right)$ by $(9)$, there must exist a time $t_{0}<L_{1}$ so that $F_{2}^{\prime}\left(t_{0}\right)=F_{1}^{\prime}\left(t_{0}\right)$, while $F_{2}^{\prime}(s)>F_{1}^{\prime}(s)$ for $s \in\left(t_{0}, L_{1}\right]$. By integration, it follows that

$$
F_{2}(s)-F_{2}\left(t_{0}\right) \geq F_{1}(s)-F_{1}\left(t_{0}\right), \quad s \in\left[t_{0}, L_{1}\right] .
$$

Combined with (38), we may apply Lemma $6\left(\right.$ iii) to deduce that $g\left(t_{0}, F_{2}\right)<$ $g\left(t_{0}, F_{1}\right)$, hence $F_{2}^{\prime}\left(t_{0}\right)>F_{1}^{\prime}\left(t_{0}\right)$. But this contradicts the definition of $t_{0}$. We have thus verified that $F_{2}^{\prime}(t)>F_{1}^{\prime}(t)$ for all $t<L$. But this implies that $F_{2}(0)-$ $F_{1}(0)<F_{2}\left(L_{1}\right)-F_{1}\left(L_{1}\right)<0$, where the first inequality follows by integration, and the second from (37).

We have thus shown that $L_{1}<L_{2}$ implies $F_{2}(0)<F_{1}(0)$, which completes the proof of Theorem 2 . 